Check for updates

Cite this: RSC Adv., 2017, 7, 50912

Received 18th August 2017

Accepted 11th October 2017

DOI: 10.1039/c7ra09161c

rsc.li/rsc-advances

\title{
Synthesis of a $\mathrm{CO}_{3} \mathrm{O}_{4}$ agold/MWCNT/polypyrrole hybrid composite for DMMP detection in chemical sensors
}

\author{
Sivalingam Ramesh, (D) ${ }^{a}$ Young-Jun Lee, ${ }^{\mathrm{b}}$ Sabeur Msolli, ${ }^{a}$ Jong-Gyu Kim, ${ }^{\mathrm{b}}$ \\ Heung Soo Kim ${ }^{\star a}$ and Joo-Hyung Kim ${ }^{\star b}$
}

\begin{abstract}
Chemical sensors based on a nanocrystalline $\mathrm{CO}_{3} \mathrm{O}_{4}$ /gold@MWCNT/polypyrrole decorated hybrid composite were fabricated, and their sensitivity properties to stimulant dimethylmethylphosphonate (DMMP) were characterized. The hybrid composite was decorated by a hydrothermal process in the presence of cobalt/gold precursors, and the characteristics were determined by Raman, XRD, XPS, SEMEDX, and FE-TEM analysis. The cyclability and linearity of the detection response for chemical sensors in the presence of DMMP vapor were determined by using a crystal microbalance. The hybrid composite material should possess excellent selectivity, stability, and reasonable sensitivity in the presence of $\mathrm{N}_{2}$ gas at room temperature for DMMP detection. The response of the composite showed retainability when exposed to the DMMP component, due to the high resistance response, fast response time, rapid recovery, and good reproducibility.
\end{abstract}

\section{Introduction}

Conductive polymers and their composites have emerged as new materials of great potential considering their cost-effective processability, light weight, tunable mechanical and magnetic properties, and environmental friendliness. Polypyrrole (PPy) has received more attention from current research due to its conductive nature, easy oxidation, water solubility, and the commercial availability of pyrrole monomers. The PPy based materials are used in the field of electro catalysts, anticorrosion, carbon dioxide capture, nanofiltration membranes for separating organic mixtures, light emitting diodes, electrochromic devices, batteries, electrochemical capacitors, and sensors. ${ }^{1,2}$ Carbon nanotubes (CNTs) have been considered as attractive candidates for sensing materials, due to their nanoscale size, with high aspect ratio, large specific surface area, good chemical stability, and excellent mechanical and electronic properties. $^{1-4}$ Therefore, CNT-based gas sensors generally possess a rapid response time, high sensitivity, low operating temperature, and small size for nanodevice miniaturization.

In addition, the development of the PPy/metal oxide hybrid composite into a low cost, highly sensitive and selective, reproducible, and low power gas sensor is always a hot topic for research, because of the potential applications in different areas, such as environmental sensors, and the detection of

\footnotetext{
${ }^{a}$ Department of Mechanical Robotics and Energy Engineering, Dongguk University-Seoul, Seoul, 04620, South Korea.E-mail: heungsoo@dgu.edu

${ }^{b}$ Department of Mechanical Engineering, Inha University, Incheon, 22212, South Korea. E-mail: joohyung.kim@inha.ac.kr
}

chemical warfare agents. ${ }^{1-5}$ Furthermore, dimethyl methylphosphonate (DMMP) is a simulant for the nerve agent sarin, and various types of sensors have been devised to detect the nerve agent, including surface acoustic wave (SAW), quartz crystal microbalance (QCM), and semiconducting metal oxide (SMO) sensors used elsewhere., ${ }^{5,6}$ The single and multiwall carbon nanotube sensor are ordered with alternating-current dielectrophoresis, and showed sensitivity to dimethyl methylphosphonate (DMMP) and thionyl chloride materials. ${ }^{7,8}$ Recent papers concerning the detection of CWAs and more precisely DMMP using metal oxide-based gas sensors observed that these kind of sensors show very interesting detection sensitivity. ${ }^{9}$ Among the transition metal oxides, cobalt oxide $\left(\mathrm{Co}_{3} \mathrm{O}_{4}\right)$, which has low cost, and an environmentally friendly nature, as well as intriguing electronic, optical, electrochemical, and electro catalytic properties, has shown great potential in the applications of supercapacitors, catalysts, and electrochemical sensors. ${ }^{9,10}$ The metals such as $\mathrm{Au}, \mathrm{Pt}, \mathrm{Pd}, \mathrm{Ni}$, and Ir are selective active phases for $-\mathrm{NO}_{2}$ group hydrogenation under mild temperature conditions in presence of metallic gold nanoparticles are reduce $\mathrm{Au}^{3+}$ cations to gold nanoparticles. ${ }^{11-13}$ Gold nanoparticles with sizes between $3-10 \mathrm{~nm}$ were firmly supported on pristine multiwalled carbon nanotubes (MWCNT) in high yields by a facile linker-free deposition route using aqueous suspensions of pre-reduced gold precursor for catalytic applications. The carbon nanotubes with containing high surface area, high chemical stability, unique electronic properties and mechanical robustness provide an effective deposition of catalytically active metal nanoparticles. Since the pioneering discovery of Haruta that gold in the form of 
nanoparticles can promote catalytic reactions in the of gold catalysis has become a discipline of increasing interest. Today, several key synthetic transformations such as oxidation and hydrogenation reactions, among others, have been successfully developed. The attachment of gold nanoparticles (NPs) to the sidewalls of CNTs is particularly promising for novel, highly efficient photo electrochemical cells and sensor devices. In the past few years, various approaches and strategies, including the self-assembly of nanoparticles on the functionalized CNTs by small molecules. The controllable loading of gold nanoparticles on carbon nanotubes can be tuned by regenerative ion exchange and subsequent reduction. This method is not limited to gold, however, it may be used to assemble a variety of other metal nanoparticles on surfaces of the modified carbon nanotubes. The hybrids show characteristic plasmon absorption of gold nanoparticles in the UV-visible spectrum and very good electrochemical properties. The results imply that these nanohybrid may have a good application potential in catalysis, sensor, and fuel cells. The uniform nanostructure of gold nanoparticles facilitate the precise correlation of structure morphology for catalytic properties. These well-defined nanocatalysts are expected to provide exciting opportunities for catalysis research and they have great potential in real applications. The metallic nanoparticles deposition from water based colloid on to solid substrates by using electrospray technique. The gold nanoparticles with the size of $10-20 \mathrm{~nm}$ were synthesized in water in presence of chemical reduction method and characterized by UV-vis spectroscopy, dynamic light scattering (DLS), and atomic force microscopy (AFM). In particular, the gold core in the nanocomposite could serve as high-performance catalyst, and the ligand shell can act as good surfactant to facilitate the dispersion of CNTs in water, as well as to provide good protection for gold core to avoid the agglomeration. The high aspect-ratio of CNTs can then be readily fabricated into a homogeneous 3D self-support membrane in presence of vacuum filtration, with a uniform distribution of nanoparticles in the membrane network. The effects of several key experimental parameters on the catalytic performance were also systematically proved the good design of gold nanocatalysts in the catalytic membrane system. The gold particles on high surface area substrate are highly desirable for various heterogeneous catalytic reactions. The numerous studies have reported that the catalytic reactivity of gold nano particles is highly size dependent, especially when the particles are in the nanoscale size range, where the physicochemical properties of gold nanoparticles are distinctively different from their bulk counterparts. Here important references are shown below and incorporated in the revised manuscript. ${ }^{14-17}$

The semiconducting metal oxides (SMOs) with hybrid composites have been used for the detection of toxic gases and vapors. Among the various SMOs, cobalt oxides, gold, $\mathrm{ZnO}, \mathrm{TiO}_{2}$, and $\mathrm{SnO}_{2}$ are the most well-defined transducers are used in the gas sensor properties. The metal oxides have been used as one component of composite materials for detecting toxic gases and vapors in the DMMP detection. Furthermore, the important to reveal the sensing mechanism of metal oxide gas sensors, which is great helpful for designing and synthesis of novel gas sensing materials with excellent performance. The fundamental sensing mechanism of metal-oxide-based gas sensors is mainly depends on the shift of equilibrium of the surface chemisorbed oxygen reaction in presence of target gas. The metal oxide semiconductors can be divided into n- and p-type, which shows different sensing properties of detecting gas. Because of the exposure to oxidizing gases are acts as acceptors due to the, resistance increases for n-type semiconductors and a decrease for p-type semiconductors. The present sensing mechanism based on metal-oxide semiconductors is mainly described by two different ways. One is the ionosorption way, and another is the oxygen-vacancy mechanism. The first way to consider the space charge effects or the changes in the electric surface of potential results from the gas adsorption, ionization and redox reactions. The second way of mechanism on the reaction between oxygen vacancies and gas molecules and the different amount of the subsurface/surface oxygen vacancies and their reduction-reoxidation process leads to changes in the oxygen stoichiometry. ${ }^{18-20}$

DMMP has also become a significant environmental and food chain pollutant, due to its large consumption as a common additive for anti-foaming agents, plasticizers, stabilizers, textile conditioners, and antistatic agents. Fig. 1 shows a gas controller system to flow the DMMP vapor. The hybrid composites were dispersed in ethanol, and sonicated for $3 \mathrm{~h}$ to get a homogeneous solution. The calculated amount of $5 \mu \mathrm{l}$ of the resultant solution was dropped in the AT-cut $5 \mathrm{MHz}$ quartz crystal via a drop coating process, and then dried in vacuum oven at $80^{\circ} \mathrm{C}$ for $60 \mathrm{~min}$, and finally the DMMP sensitivity and selectivity were measured (Fig. 1) shows a schematic representation of the dropping process in detail. The system consists of valves, bubbler, steel tube, and two mass flow controllers (MFC) (KOFLOG, Japan). The frequency shifts induced by absorption were monitored by using a QCM 200 Digital Controller (Stanford Research System, SRS). The collected data from the controller were transferred to a computer through a GPIB interface. A QCM 25 Crystal Oscillator (Stanford Research System, SRS) was used to excite the QCM sensor. Concentrated vapor was modulated by regulating the flow rate of carrier gases using two MFCs. Nitrogen gas was used for a carrier and dilution. The output flow rate of saturated solvent vapor $\left(F_{\mathrm{SV}}\right)$ can be calculated by the following equation:

$$
F_{\mathrm{SV}}=\left(\frac{P_{\mathrm{S}}}{P_{0}-P_{\mathrm{S}}}\right) F_{\mathrm{C}}
$$

where, $F_{\mathrm{C}}(\mathrm{sccm})$ is the carrier flow rate, $P_{0}$ is the pressure in outlet bubbler, and $P_{\mathrm{S}}(\mathrm{mmHg})$ is the vapor pressure of DMMP. ${ }^{11-17}$

$$
P_{\text {DMMP }}=2.844 \times 10^{8} \times \exp \left(\frac{-11500}{R T}\right)
$$

where, $T$ is the bubbler temperature of DMMP, and $R$ is a gas constant. The saturated DMMP and diluted nitrogen gas were mixed, and delivered to the sensor. The resultant DMMP concentration, $C_{\mathrm{DMMP}}(\mathrm{ppm})$, was calculated by using:

$$
C_{\text {DMMP }}(\mathrm{ppm})=\frac{10^{6} F_{\mathrm{DMMP}}}{F_{\mathrm{D}}+F_{\mathrm{C}}+F_{\mathrm{DMMP}}}
$$




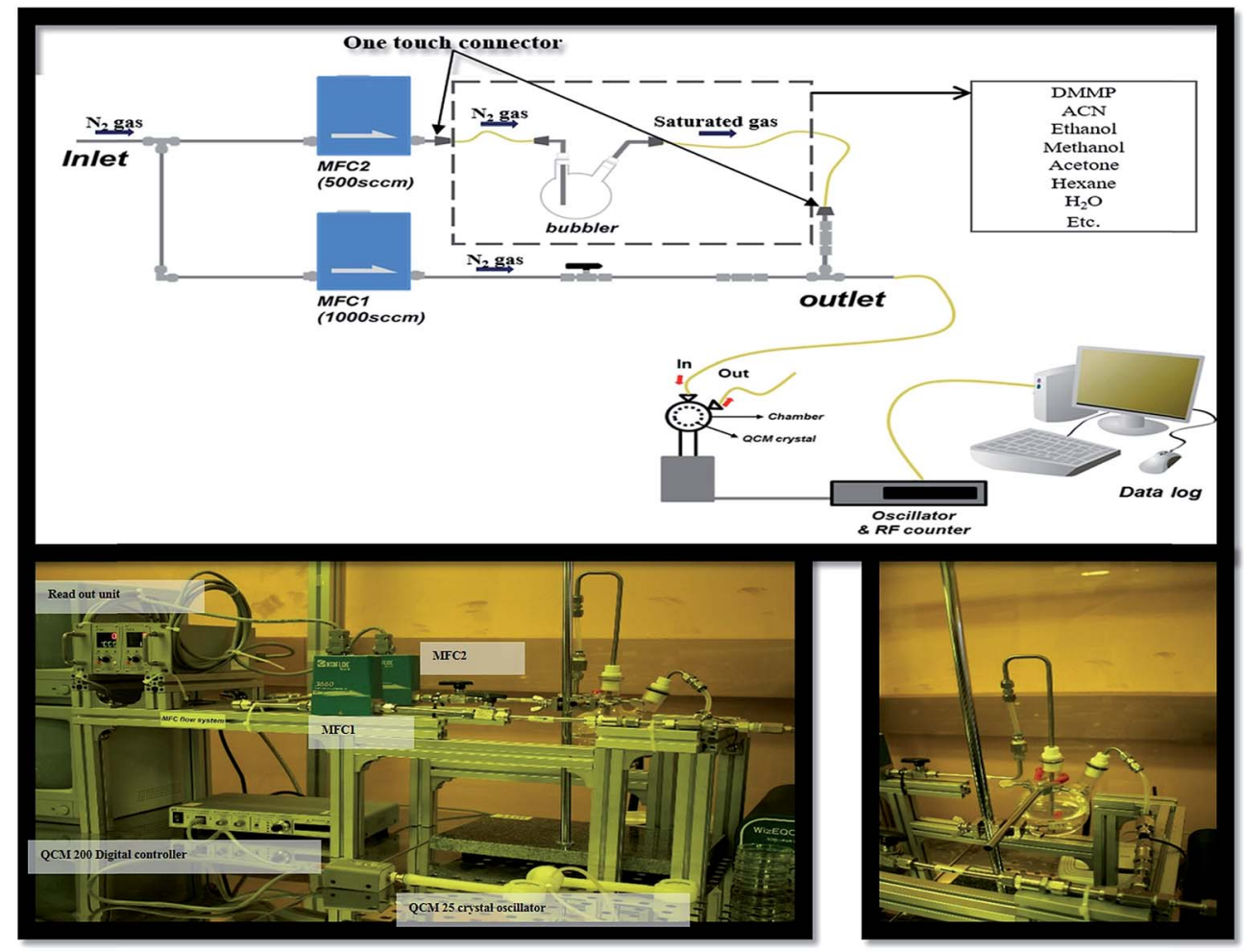

Fig. 1 A photographs of the feeding system connected to the QCM.

where, $F_{\mathrm{D}}$ is the flow rate of the dilution gas, which is fixed at $1000 \mathrm{sccm}$. All the experiments were carried out at room temperature.

The concentration of DMMP vapor was modulated by regulating the flow rate of carrier gas using two mass flow controllers in the presence of $\mathrm{N}_{2}$ gas. The DMMP vapor was generated by a bubbler system. The output flow rate of DMMP vapor can be calculated using a bubbler and the Antoine equation. In the present study focused on the nanocrystalline $\mathrm{Co}_{3} \mathrm{O}_{4}$ / gold@MWCNT/PPy decorated hybrid composite, network sensors were fabricated, and their DMMP gas sensing characteristics were characterized. The fabricated network sensors stably detected the ppm level of DMMP with a fast response time, even at room temperature, by cooperative combination of cobalt/gold and MWCNT/PPy.

\section{Experimental}

\subsection{Synthesis of carboxylic group's functionalization of MWCNTs}

The oxidation of multiwall carbon nanotubes (MWCNTs) was synthesized according to the reported procedure. ${ }^{\mathbf{2 1}}$ The calculated amount of $2 \mathrm{~g}$ of MWCNTs was dispersed in $100 \mathrm{ml}$ of concentrated $\mathrm{H}_{2} \mathrm{SO}_{4}$ and $\mathrm{HNO}_{3}$ with a volume ratio of $3: 1 \mathrm{using}$ an ultrasonicator for $1 \mathrm{~h}$. The reaction mixture was heated at $90{ }^{\circ} \mathrm{C}$ for $7 \mathrm{~h}$, and washed with water several times, until becoming a neutral $\mathrm{pH}=7$. The resultant product of functionalized MWCNTs was filtered by using a $0.2 \mu \mathrm{m}$ PTFE membrane filter, and dried in vacuum at $80{ }^{\circ} \mathrm{C}$ for $24 \mathrm{~h}$.

\subsection{Synthesis of $\mathrm{Co}_{3} \mathrm{O}_{4} /$ gold@MWCNT/polypyrrole (PPy) hybrid composite}

The calculated amount of functionalized MWCNTs (0.25 g) was dispersed in $50 \mathrm{ml}$ of water, and $0.9 \mathrm{~g}$ of urea/ammonia base (pH-9) was added, and stirred for $4 \mathrm{~h}$. The required amount of $2.9 \mathrm{~g}$ of cobalt chloride was added, and stirred for $12 \mathrm{~h}$ at $90^{\circ} \mathrm{C}$. In addition, the required amount of $0.5 \mathrm{~g}$ of PPy-MWCNT, and $7.25 \mathrm{~g}$ of cobalt chloride was dissolved in $50 \mathrm{ml}$ of distilled water, with vigorous stirring at $90{ }^{\circ} \mathrm{C}$ for $3 \mathrm{~h}$. The PPy-MWCNT and cobalt chloride were mixed together, and were well dispersed in the presence of water. The reaction mixture becomes a strong green colour, due to the presence of cobalt oxide, and then $0.02 \mathrm{~g}$ of gold chloride was added, and reduction occurred, followed by a catalytic amount of formic acid. The resultant product was collected, and several times repetitively purified by ethanol. After that, calcination of the product of hybrid composite was conducted at $200{ }^{\circ} \mathrm{C}$ for $12 \mathrm{~h}$. Furthermore, the hybrid composite was coated on quartz crystal by a drop coating process in the presence of ethanol, and sonicated for $2 \mathrm{~h}$. The resultant solution was transparent, and then $5 \mu \mathrm{l}$ was dropped on the crystal in the coating process.

\subsection{Materials characterization}

All chemicals reagents were of analytical grade, and were used without any further purification process. Pyrrole, MWCNT, sulphuric acid, nitric acid, ferric chloride $\left(\mathrm{FeCl}_{3}\right)$, sodium dodecyl benzenesulfonate (SDBS), butanol, hexane and cobalt chloride, gold chloride, formic acid and solvents were used in 

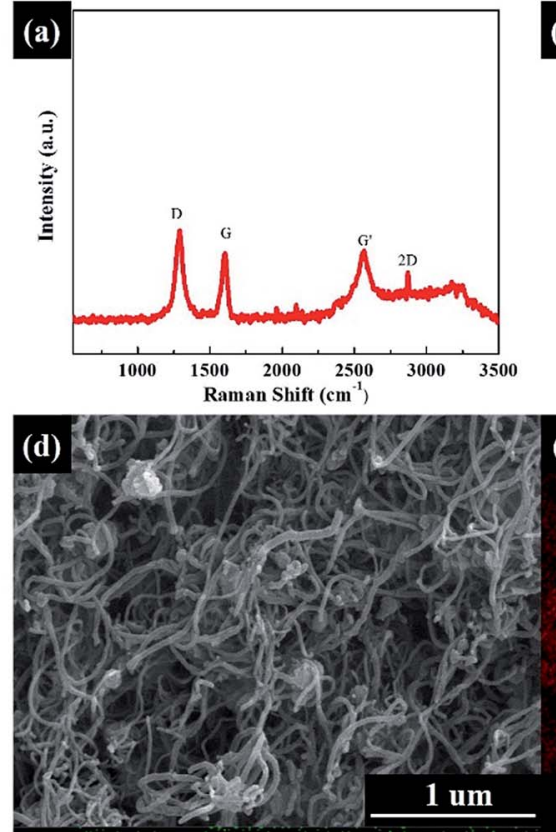

(g)

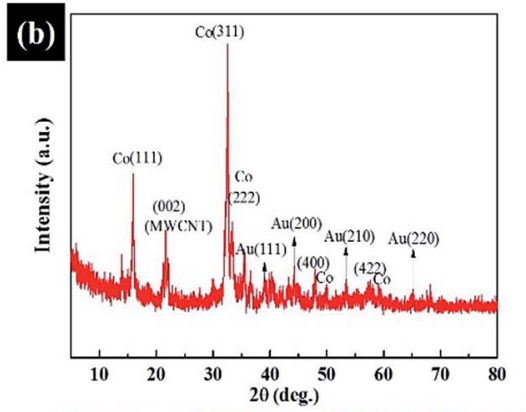

(e)

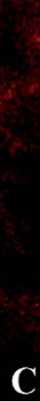

(h)

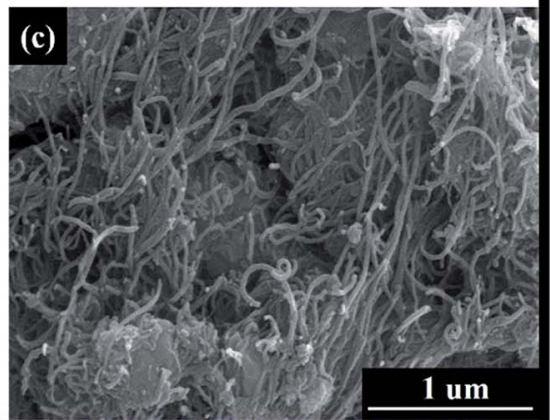

(f)

$\mathbf{0}$

(i)

Fig. 2 (a) Raman spectrum, (b) XRD patterns, (c) and (d) SEM morphology, and (e)-(i) EDS mapping of the hybrid composite.

the present study as received from Aldrich Chemicals Company, South Korea. Morphological and selected-area electron diffraction (SAED) images were obtained by high-resolution transmission electron microscopy (HRTEM, JEOL 2010F) equipped with an energy-dispersive X-ray spectrometer (EDS). The surface properties were analyzed by X-ray photoelectron spectroscopy (XPS, Thermo Scientific, K-Alpha) using Al K $\alpha$ radiation. Raman spectroscopy was performed on a Raman microscope (UniRAM, UniNanoTech., Korea). The crystallinity of the sample was examined by X-ray diffraction (XRD, X'Pert PRO MRD) using Cu$\mathrm{K} \alpha$ radiation. Frequency shifts of hybrid composite coated on QCM sensor were observed by QCM 200 digital controller, and a QCM 25 Crystal Oscillator (Stanford Research System, SRS) was used to excite the QCM quartz (AT-cut $5 \mathrm{MHz}$ quartz crystal) with $\mathrm{Cr} / \mathrm{Ag}$ electrode on both sides (Fig. 1).

\section{Results and discussions}

The structural and electronic properties of the hybrid composite were studied by Raman spectroscopy. Previous reports have shown the spectral results of MWCNT and cobalt/Au-carbon composites. ${ }^{22}$ Fig. 2 shows the results, which reveal two characteristic peaks located at 1284 and $1608 \mathrm{~cm}^{-1}$ from MWCNT, which can be attributed to $\mathrm{D}$ and $\mathrm{G}$ bands, respectively. The $\mathrm{D}$ bands correspond to a defect induced in the $\mathrm{A}_{1 \mathrm{~g}}$ plane zone-edge, and the $\mathrm{G}$ band is attributed to $\mathrm{E}_{2 \mathrm{~g}}$ modes. This confirms the interaction between $\mathrm{Co}^{2+} / \mathrm{Au}$ nanoparticles in the MWCNT surface, and accords with the reported literature. ${ }^{22}$

X-ray diffraction peaks (Fig. 2(b)) show at $13.90^{\circ}, 15.91^{\circ}$, $21.68^{\circ}, 29.85^{\circ}, 32.63^{\circ}, 33.40^{\circ}, 35.32^{\circ}, 36.64^{\circ}, 39.03^{\circ}, 40.49^{\circ}$, $44.34^{\circ}, 47.81^{\circ}, 50.06^{\circ}, 53.53^{\circ}, 57.65^{\circ}, 59.29^{\circ}, 65.18^{\circ}$, and $66.39^{\circ}$, which can be respectively denoted as (111), (220), (311), (222), (400), (422), (511), (440), and (533) reflections of the facecentered cubic structure of $\mathrm{Co}_{3} \mathrm{O}_{4}$ (JCPDS no. 43-1003), and the diffraction peaks can be indexed as the (111), (200), (220), (311), and (222) reflections of the face-centered cubic (fcc) structure of $\mathrm{Au}$ (JCPDS 04-0784). The peak at $27.79^{\circ}$ corresponds to the (002) reflection of MWCNT. ${ }^{22}$ Fig. 2(c) and (d) indicate the SEM morphology and Fig. 2(e) and (f) the EDS mapping images of the cobalt oxide/Au in the N-doped MWCNT hybrid composite. 
(a)

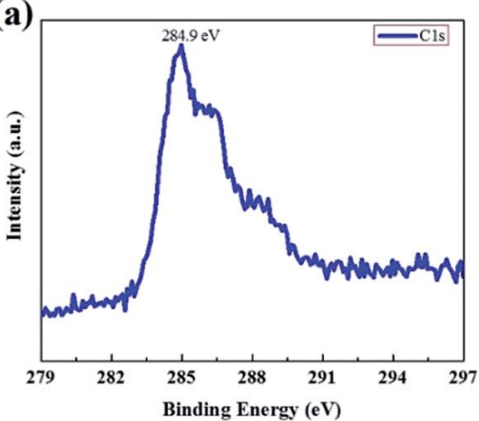

(d)

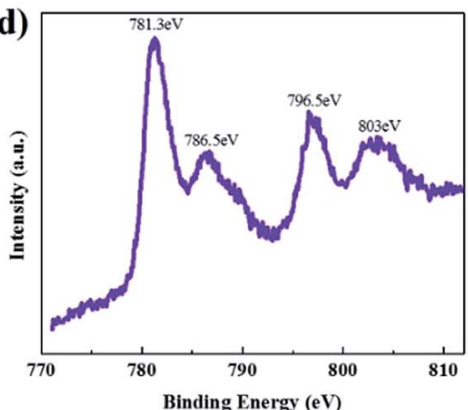

(b)

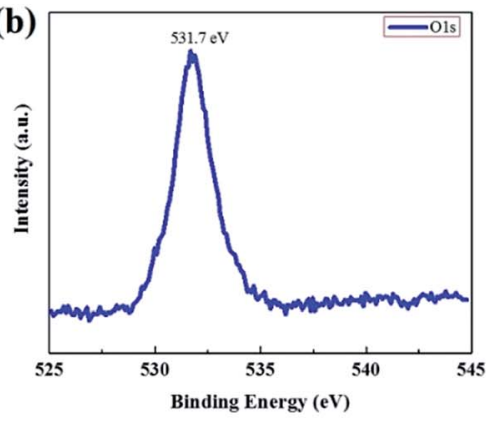

(e)

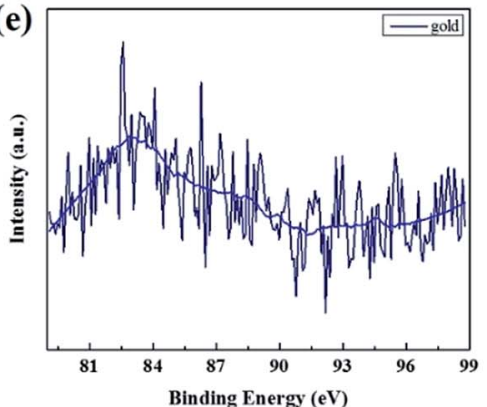

(c)

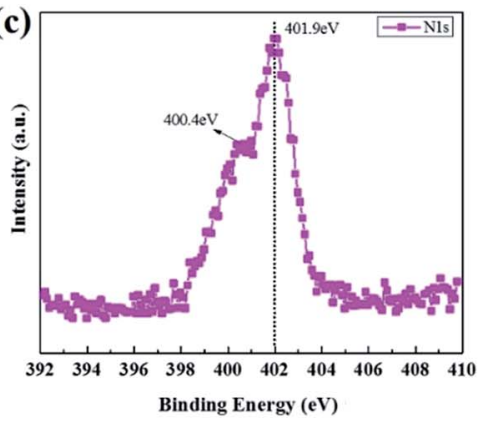

(f)

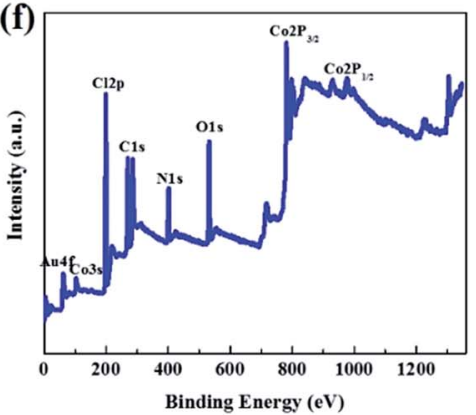

Fig. 3 XPS results of (a) C1s (b) O1s (c) N1s (d) $\mathrm{Co}_{2} \mathrm{P}_{3 / 2}$ and $\mathrm{Co}_{2} \mathrm{P}_{1 / 2}$ (e) gold (f) survey of hybrid composite.

The XPS technique becomes useful in order to further investigate the structural evolution of the as-synthesized hybrid composite. The survey XPS spectrum (Fig. 3) of the composite showed C1s (284.9), O1s (531.7), N1s (400.4-401.9), and Co2p $(781.3 \mathrm{eV})$, which confirmed the presence of these elements in the composite. The spin-orbit peaks in the high-resolution

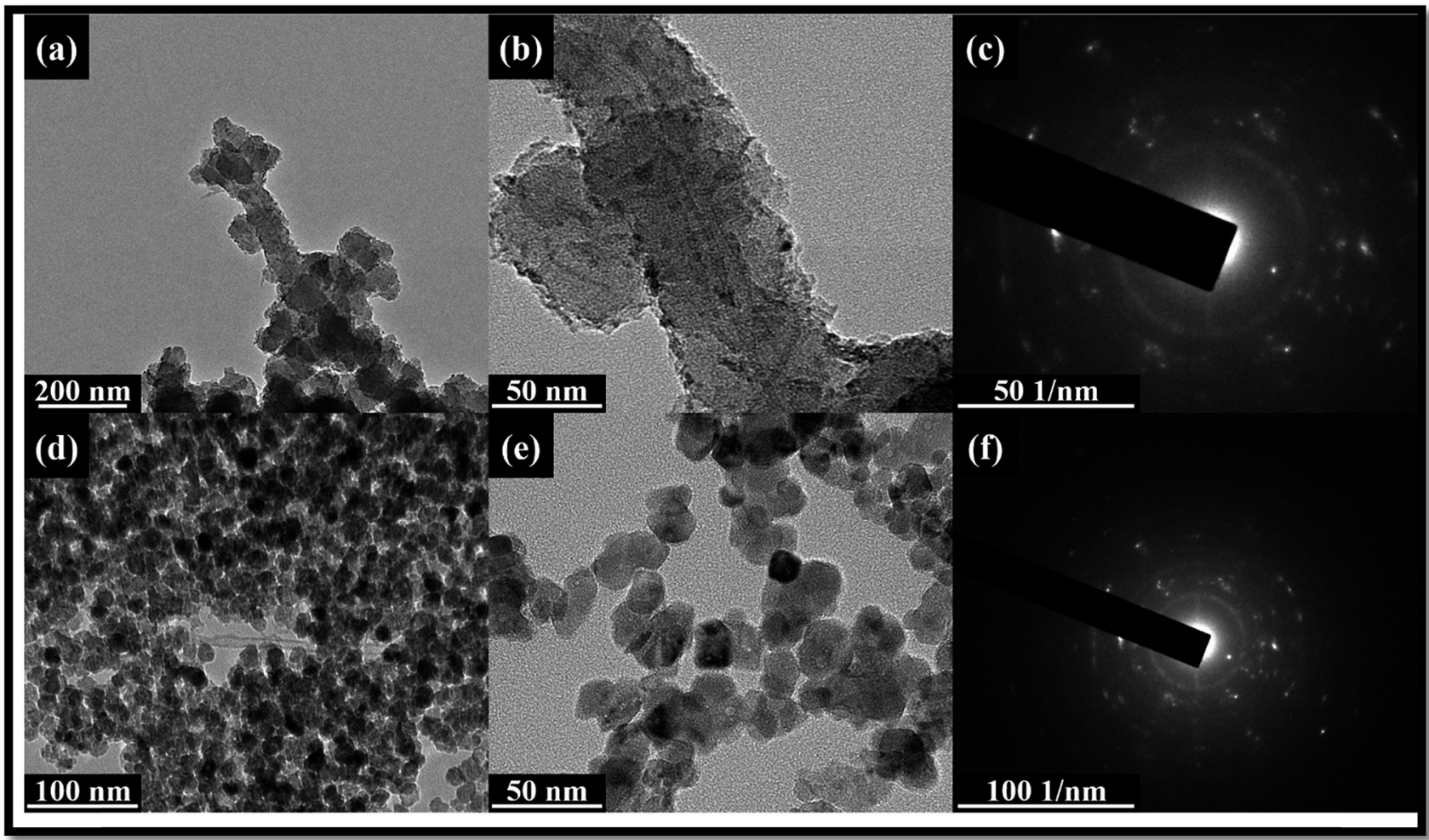

Fig. 4 (a) and (b) TEM images (different magnifications), and (c) SAED pattern, of MWCNT/PPy; and (d) and (e) TEM images, and (f) SAED pattern, of $\mathrm{CO}_{3} \mathrm{O}_{4} / \mathrm{Au}$ (MMWCNT/PPy hybrid composite. 
spectrum for Co2 $\mathrm{p}_{3 / 2}$ and Co2 $\mathrm{p}_{1 / 2}$ were observed at 786.5, 796.5, and $803 \mathrm{eV}$, respectively, which are found to be in good agreement with the previous studies. ${ }^{22,23}$ The core-level N1s spectrum showed three peaks at $400.4-401.9 \mathrm{eV}$, corresponding to pyridinic- $\mathrm{N}$, graphitic $\mathrm{N}$, and oxidized $\mathrm{N}$, respectively. The intensity of the graphitic $\mathrm{N}$ at the $401.9 \mathrm{eV}$ peak was much higher than those of the other two peaks, suggesting the dominance of PPy nitrogen. In addition, the Au4f spectrum was recorded from the hybrid composite sample, where $A u 4 f_{7 / 2}$ and $\mathrm{Au} 4 \mathrm{f}_{5 / 2}$ spin-orbit doublets were observed to be located at 83.6 and $87.3 \mathrm{eV}$, respectively, in the binding energy scale. Therefore, it is reasonable to conclude that the MWCNT was decorated with both cobalt oxides and Au hybrid composites. ${ }^{24-45}$

TEM images (Fig. 4(a)-(c)) show the MWCNT/PPy, and Fig. $4(\mathrm{~d})-(\mathrm{f})$ the $\mathrm{Co}_{3} \mathrm{O}_{4} / \mathrm{Au}$ decorated nanotubes with a mean diameter of approximately $\sim 10-20 \mathrm{~nm}$ that were dispersed on the MWCNT/PPy surface. The SAED pattern of the composite (Fig. 4(f)) showed well-defined rings, which could be attributed to the crystalline nature of $\mathrm{Co}_{3} \mathrm{O}_{4} / \mathrm{Au}^{41,44,45}$

The metal oxide gas sensors have been investigated extensively since decades ago owing to their uses of high response to pollutant gases, fast response and recovery, low cost, easy processing, and controlled particle size. However, they are usually operated at temperature range between 200 and $250^{\circ} \mathrm{C}$. Sensors based on metal oxides such as cobalt oxide, gold, $\mathrm{SnO}_{2}, \mathrm{WO}_{3}$ or $\mathrm{TiO}_{2}$ modified CNTs can detect gases of $\mathrm{NO}_{2}, \mathrm{CO}, \mathrm{NH}_{3}$ and ethanol vapors at low temperature process for improved sensing properties. Recently, sensors based on carbon based hybrid nanocomposites with enhanced gas sensing performance have been reported. Wei et al. constructed SWCNTs/metal oxides gas sensor by incorporating SWCNTs into a metal oxide substrate. The synthesis of the SWCNTs layer in presence of heat treatment process, which was fabricated by spin coating using an organometallic solution dispersed with SWCNTs. The prepared SWCNTs hybrid nanocomposite sensors exhibit much higher response and recovery property in detecting $\mathrm{NO}_{2}$ at room temperature. The response time and recovery time are 9 minutes and 1.5 minutes, respectively. This may be related to the potential barriers of electronic conduction in the hybrid composite material.

In order to explain the electrical response obtained under DMMP flow, and according to results reported in previously. ${ }^{41-47}$ We propose different reactional mechanisms, which allow the understanding of the behaviour of DMMP when arriving onto the sensor's surface. According to this mechanism, if we consider now the electrical response obtained when DMMP is detected using tin dioxide sensors, it clearly appears that the tin dioxide sensor is solely exposed to DMMP, $\mathrm{CO}_{2}$ and

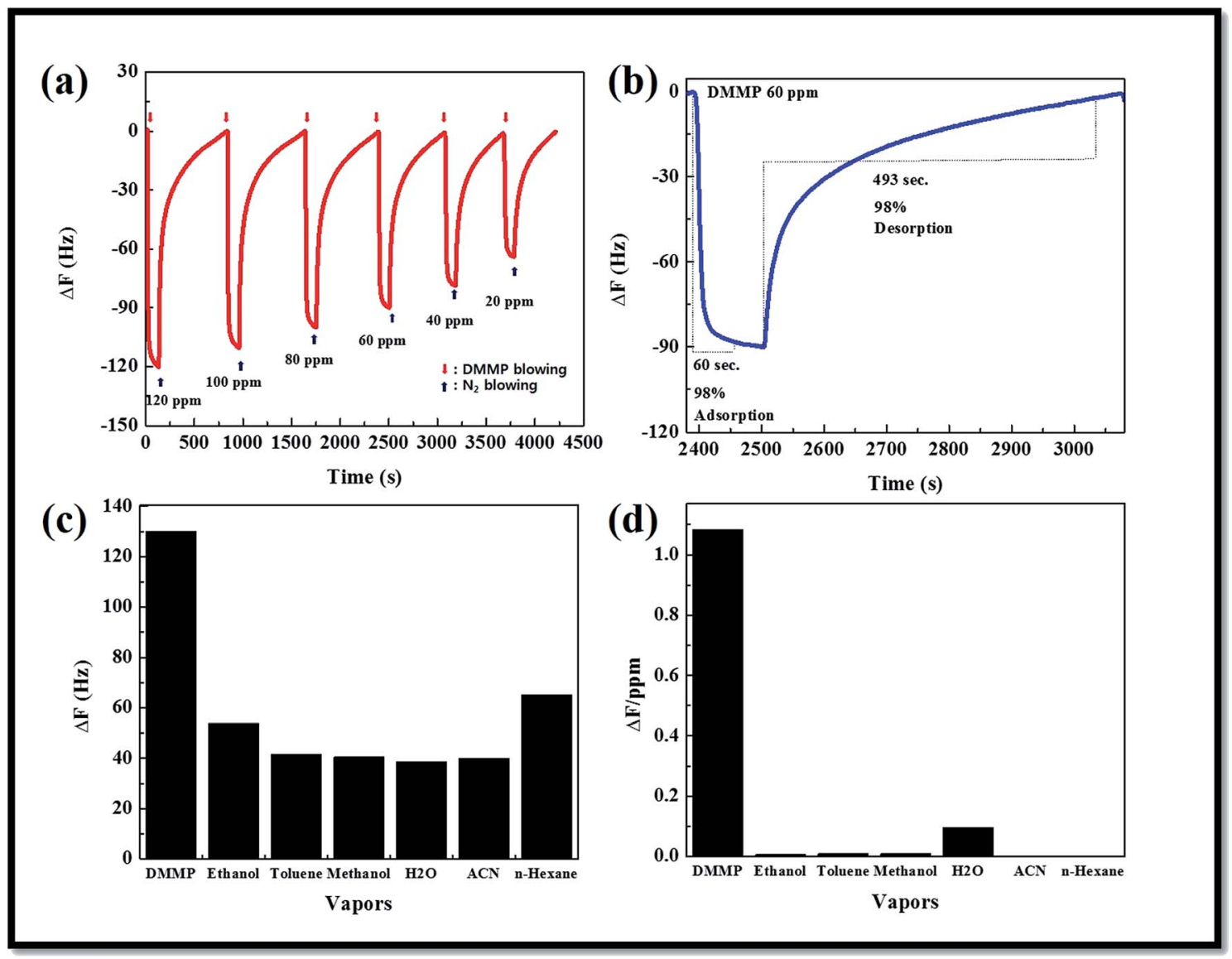

Fig. 5 (a) Frequency shift of the hybrid composite decorated sensor under 20-120 ppm of DMMP. (b) Speed of absorption and desorption. (c) Selectivity of the hybrid composite sensing material with ethanol, $\mathrm{H}_{2} \mathrm{O}$, toluene, and $\mathrm{n}$-hexane. (d) Calibration of selectivity of different solvent in ppm level of hybrid composite. 
methylphosphonic acid. As far as $\mathrm{CO}_{2}$ is concerned it is wellknown that metal oxide based gas sensors show a very weak sensitivity to this compound. It thus seems that the electrical response obtained during the detection of DMMP vapours is principally induced by the interaction of phosphorous compounds with the sensor. In order to explain the increase in conductance obtained when DMMP is detected using tin dioxide-based gas sensors, we propose a reactional mechanism which takes place in two stages. First, DMMP is adsorbed onto the metal oxide surface, allowing it to react with an oxygen species $\left(\mathrm{O}^{-}\right)$. This reaction leads to the formation of methylphosphonic acid, which remains adsorbed onto the metal oxide, $\mathrm{CO}_{2}$ which does not react with the sensor, and $\mathrm{H}_{2} \mathrm{O}$. At the same time electron can be released to the conduction band leading to an increase in the metal oxide conductance. Fig. 5(a) shows the response of the deposited sensor to DMMP vapor in the concentration of 20-120 ppm. The sensor shows that saturation for a long exposure time depends on the amount of resistance change with the increase of DMMP vapor concentration at any exposure time. The effective response time is an operational parameter for the sensor to differentiate the different amounts of DMMP concentration. In the following experiment, the sensor response $\left(R_{\mathrm{r}}\right)$ upon exposure to DMMP vapor under the effective response time is defined by equation. Fig. 5(b) shows the frequency shift of the hybrid composite QCM sensor according to DMMP concentration. As the concentration of DMMP decreased, the frequency shift decreased. The response and recovery stabilizing time were 60 and $439 \mathrm{~s}$ for $98 \%$ of the maximum frequency response to DMMP vapor, respectively. The recovery time using $\mathrm{N}_{2}$ blowing was longer than the response time. Fig. 5(c) and (d) show the selectivity of the hybrid composite QCM sensor that analyzed various vapors, such as ethanol, water, methanol, toluene, nhexane, and DMMP, under a fixed 120 ppm vapor gas. ${ }^{41-47}$

\section{Conclusions}

In summary, the nanocrystalline structure of $\mathrm{a} \mathrm{Co}_{3} \mathrm{O}_{4} / \mathrm{Au}$ decorated MWCNT/PPy hybrid composite was successfully synthesized via a hydrothermal process. The hybrid composites were characterized by Raman, XRD, XPS, SEM-EDX, and HR-TEM analysis. The different analyses show the decorated cobalt oxide/Au to be present in the MWCNT surface, and its structural and morphological properties. The FE-TEM morphology confirmed the nanocrystalline structure of cobalt oxide in the presence of MWCNT/PPy surface. Furthermore, the hybrid composite deposited on quartz via sensors exhibited an excellent response to DMMP vapor at room temperature and low power consumption at a sampling voltage of $300 \mathrm{mV}$. The enhancement can be achieved by the combination of $\mathrm{Co}_{3} \mathrm{O}_{4} / \mathrm{Au}$ nanoparticle composite that actively reacts with the gases, and the formation of an electrical continuum between $\mathrm{Co}_{3} \mathrm{O}_{4}$ and MWCNT/PPy. Therefore, the hybrid composite showed a rapid, reversible, and reproducible response to DMMP vapor, indicating its promise as a gas sensor for high-performance detection.

\section{Conflicts of interest}

There is no any conflicts of interest.

\section{Acknowledgements}

This research was supported by the Basic Science Research Program through the National Research Foundation of Korea (NRF-2017R1D1A1B03028368), funded by the Ministry of Education and Agency of Defense and Development Program (ADD CBDRC, CBD-12) of the Korean Science \& Engineering Foundation, which is funded by the Korean government (MOEHRD, Grant No. R32-2008-000-10124-0) and also supported by Global Research Development Center Program through the National Research Foundation of Korea (NRF) funded by the Ministry of Science and ICT (MSIT) (2017K1A4A3013662).

\section{References}

1 G. Sanjay Bachhav and R. D. Patil, J. Mater. Sci. Chem. Eng., 2015, 3, 30-44.

2 S. M. Jebreiil Khadem, Y. Abdi, S. Darbari and F. Ostovari, Curr. Appl. Phys, 2014, 14, 1498-1503.

3 Y. Liu, L. Wang, H. Zhang, F. Ran, P. Yang and H. Li, RSC Adv., 2017, 7, 40119-40123.

4 T. Zhang, S. Mubeen, N. V. Myung and M. A. Deshusses, Nanotechnology, 2008, 193, 32001-32014.

5 B. Renganathan, D. Sastikumar, A. Chandra Bose, R. Srinivasan and A. R. Ganesan, Curr. Appl. Phys, 2014, 14, 467-471.

6 D. Burman, R. Ghosh, S. Santra and P. K. Guha, RSC Adv., 2016, 6, 57424-57433.

7 N. D. Hoa, N. V. Quy, Y. Cho and D. Kim, Sens. Actuators, B, 2009, 135, 656-663.

8 R. L. VanderWal, G. W. Hunterb, J. C. Xu, M. J. Kulis, G. M. Berger and T. M. Ticich, Sens. Actuators, B, 2009, 138, 113-119.

9 C. X. Wang, L. W. Yin, L. Y. Zhang, D. Xiang and R. Gao, Sensors, 2010, 10, 2088-2106.

10 N. Barsan and U. Weimar, J. Electroceram., 2001, 7, 143-167. 11 M. C. Daniel and D. Astruc, Chem. Rev., 2003, 104, 293-346. 12 M. Grzelczak, J. Perez-Juste, P. Mulvaney and L. M. LizMarzan, Chem. Soc. Rev., 2008, 37, 1783-1791.

13 K. Saha, S. S. Agasti, C. Kim, X. Li and V. M. Rotello, Chem. Rev., 2012, 112, 2739-2779.

14 Y. Liu, Y. Zheng, B. Du, R. Rahman Nasaruddin, T. Chen and J. Xie, Ind. Eng. Chem. Res., 2017, 56, 2999-3007.

15 J. Fang, B. Zhang, Q. Yao, Y. Yang, J. Xie and N. Yan, Coord. Chem. Rev., 2016, 322, 1-29.

16 K. Soliwoda, M. Rosowski, E. Tomaszewska, B. TkaczSzczesna, G. Celichowski and J. Grobelny, Colloids Surf., A, 2015, 486, 211-217.

17 Y. Kobayashi, Y. Tadaki, D. Nagao and M. Konno, J. Colloid Interface Sci., 2005, 283, 601-604.

18 J. Huang and Q. Wan, Sensors, 2009, 9, 9903-9924. 
19 J. S. Park, X. P. Shen and G. X. Wang, Sens. Actuators, B, 2009, 136, 494-498.

20 Y. Jia, L. F. He, Z. Guo, X. Chen, F. L. Meng, T. Luo, M. Q. Li and J. H. Liu, J. Phys. Chem. C, 2009, 113, 9581-9587.

21 W. K. Jang, J. Yun, H. I. Kim and Y. S. Lee, Carbon Lett., 2011, 12, 162-166.

22 J. S. Park, X. P. Shen and G. X. Wang, Sens. Actuators, B, 2009, 136, 494-498.

23 G. Korotcenkov, Sens. Actuators, B, 2005, 107, 209-232.

24 E. Brunol, F. Berger, M. Fromm and R. Planade, Sens. Actuators, B, 2006, 120, 35-41.

25 Z. Ying, Y. Jiang, X. Du, G. Xie, J. Yu and H. Wang, Sens. Actuators, B, 2007, 125, 167-172.

26 E. Comini, G. Faglia, G. Sberveglieri, Z. W. Pan and Z. L. Wang, Appl. Phys. Lett., 2002, 81, 1869-1871.

27 B. A. Blajeni and M. M. Boucher, Langmuir, 1989, 5, 170-174. 28 V. M. Bermudez, J. Phys. Chem. C, 2007, 111, 3719-3728.

29 V. M. Bermudez, J. Phys. Chem. C, 2009, 113, 1917-1930.

30 D. A. Trubitsyn and A. V. Vorontsov, J. Phys. Chem. B, 2005, 109, 21884-21892.

31 C. N. Rusu and J. T. Yates, J. Phys. Chem. B, 2000, 104, 1229212298.

32 M. B. Mitchell, V. N. Sheinker and E. A. Mintz, J. Phys. Chem. $B, 1997,101,11192-11203$.

33 T. M. Tesfai, V. N. Sheinker and M. B. Mitchell, J. Phys. Chem. B, 1998, 102, 7299-7302.
34 M. B. Mitchell, V. N. Sheinker and W. W. Cox, J. Phys. Chem. C, 2007, 111, 9417-9426.

35 K. D. Schierbaum, Sens. Actuators, B, 1995, 24, 239-247.

36 E. Comini, M. Ferroni, V. Guidi, G. Faglia, G. Martinelli and G. Sberveglieri, Sens. Actuators, B, 2002, 84, 26-32.

37 A. A. Tomchenko, G. P. Harmer and B. T. Marquis, Sens. Actuators, B, 2005, 108, 41-55.

38 N. J. Choi, Y. S. Lee, J. H. Kwak, J. S. Park, K. B. Park, K. S. Shin, H. D. Park, J. C. Kim, J. S Huh and D. D. Lee, Sens. Actuators, B, 2005, 108, 177-183.

39 H. Yoon and J. Jang, Adv. Funct. Mater., 2009, 19, 1567-1576. 40 N. Barsan and U. Weimar, J. Electroceram., 2001, 7, 143-167.

41 D. Barreca, E. Comini, A. Gasparotto, C. Maccato, A. Pozza, C. Sada, et al., J. Nanosci. Nanotechnol., 2010, 10, 8054-8061.

42 L. Usman and L. Franz Dickert, Sensors, 2015, 15, 3050430524.

43 H. M. Duan, D. Y. Xu, W. Z. Li and H. Y. Xu, Catal. Lett., 2008, 124, 318-323.

44 L. Y. Chen, T. Fujita, Y. Ding and M. W. Chen, Adv. Funct. Mater., 2010, 20, 2279-2285.

45 S. Sahoo, G. Karthikeyan, G. C. Nayak and C. K. Das, Synth. Met., 2011, 161, 1713-1719.

46 Y. J. Yu, C. Ouyang, Y. Gao, Z. H. Si, W. Chen, Z. Q. Wang and G. Xue, J. Polym. Sci., Part A: Polym. Chem., 2005, 43, 61056115.

47 S. Sharma, S. Hussain, S. Singh and S. Islam, Sens. Actuators, $B, 2014,194,213-219$. 